\title{
Interactive comment on "Characterization of Transport Regimes and the Polar Dome during Arctic Spring and Summer using in-situ Aircraft Measurements” by Heiko Bozem et al.
}

\section{Anonymous Referee \#1}

Received and published: 6 March 2019

This manuscript provides an analysis of air masses present during the 2014 and 2015 NETCARE airborne measurements spanning a broad region of the western Arctic between Spitsbergen and Alaska. An overview of the meteorological conditions during the summer (2014) and winter/spring (2015) campaigns is given, then trajectories are used to identify airmass history. Trace gas observations are described, then combined with potential temperature to identify regions with sharp gradients in $\mathrm{CO}$ and CO2. These gradients are used to define the "polar dome", the region of the cold, stable, near-surface Arctic airmass that is most isolated from midlatitude influences. (As this airmass wobbles over sources of pollution in the winter, it accumulates pollutants because sinks are very slow, leading to the seasonal near-surface "Arctic haze"

Printer-friendly version

Discussion paper 
phenomenon, which is of broad interest.) The statistics of $\mathrm{CO}$ and $\mathrm{CO} 2$ abundance in the different regions identified from this analysis are then presented. Next, a "transport regime" analysis, based on the trajectories and using methods developed in Binder (2017), is used to evaluate the influence of lifting within and outside the Arctic, and diabatic processes, on $\mathrm{CO}$ and $\mathrm{CO} 2$ mixing ratios within and outside of the polar dome. Finally, a discussion section evaluates the abundance of nucleation and Aitken mode particles within the three regimes identified in the earlier analysis (inside the polar dome, outside the polar dome, and a mixing region).

This is an ambitious manuscript, with many parts. It has interesting sections, but it doesn't seem to have a strong overall purpose. My fundamental complaint with the manuscript is that it doesn't make the case for any generality to the analysis. Are the results more broadly applicable outside of the narrow time period and location of the NETCARE airborne observations in 2014 and 2015? For example, in Sect. 5.3 there is a long discussion of how the $\mathrm{CO}$ and $\mathrm{CO} 2$ observations can be used to identify the polar dome boundaries, and a specific range of potential temperatures and latitudes is the result of the analysis. This is great for these NETCARE observations, but are these findings more broadly applicable? For example, could one take the long-term surface observations at UtqiaÄąvik (Barrow) or Alert or Zeppelin, apply the potential temperature and $\mathrm{CO} / \mathrm{CO} 2$ screens developed in this manuscript, and separate the data out into "in the polar dome" and "out of the polar dome" datasets? This would be useful to the scientific community. Without such broader relevance, this analysis is of interest only to the very small set of scientists interested in the NETCARE data.

In addition to my concern with the applicability of the findings, I feel the manuscript also needs restructuring. The paper opens with the meteorological analysis, which is fine. Next, though, is the trajectory analysis. It would be more logical if the next section were the identification of the polar dome using the trace gas gradients. Then the backtrajectories could follow, with backtrajectories initiated either within the polar dome, outside of it, or in the mixing region. The trajectories would then provide an independent and

Printer-friendly version

Discussion paper
Interactive comment 
intuitive confirmation of the identification of the polar dome that was derived from the trace gas and potential temperature data. Section 5.4, which is a presentation of PDFs of $\mathrm{CO}$ and $\mathrm{CO} 2$ from the three different airmasses is not very logical. $\mathrm{CO}$ and $\mathrm{CO} 2$ were used to identify the three airmass classifications, after all, so it's entirely expected that they would have different PDFs-the reasoning is circular. Instead of this section, the next logical section would be examining the transport regimes using the Binder et al. methodology, as it continues the analysis of trajectories. This section could be made stronger by coupling the delta-theta/delta-T plots with graphs of trajectory clusters (e.g., plots trajectory altitudes as a function of latitude). This would bolster the rather speculative discussion about the meaning of each of the sectors of the Binder plots. This section could get rather long, so it might make sense to give one example (from the springtime flights, perhaps) and place the rest in the supporting materials. This analysis is the part of the current manuscript that is really informative outside of the narrow range of the NETCARE project, as it suggest broader generalities about how transport occurs into the polar dome. l'd like to see it developed more thoroughly and the conclusions made firmer, perhaps with a concluding paragraph that summarizes the findings from this section.

Following the transport regime analysis, the next logical section would be to see how these different airmass types are manifested in the pollution loadings. This is currently Sect. 6, which is labelled "discussion". I appreciate the novelty of Fig. 15, but it's very difficult to understand the grey-scale coloring on top of the colored classification scheme. I'd much rather see PDFs of the number concentrations in the 5-20 and 20100 and $>100$ size class. Are there also observations of aerosol composition (e.g., $\mathrm{BC}$ abundance, composition) that could be added to this section? You've effectively classified the measurements into airmass type; it would be extremely interesting to see how all the available aerosol microphysical and chemical parameters vary within these different airmasses, and compare them with existing literature values.

Printer-friendly version

Finally, if it's feasible it would be wonderful to extend this analysis to the surface data 
from the long-term monitoring sites. This would show that the classifications developed here are more broadly applicable. At least an evaluation of whether the approach here is applicable to other cases, or is specific to NETCARE, is needed.

In addition to these larger, structural issues, the manuscript needs some technical correction. The primary author is not a native English speaker, but English-speaking co-authors should step up and give the manuscript a round of thorough copy-editing. Verb tense is not used consistently, which is distracting and sometimes confusing. (Example, p. 17 lines 18-21 go from "now calculated" to "determine" to "finally used".) There are quite a few typos that a spell checker should find, and terms like "surfacenear" instead of "near-surface" are present.

This manuscript has a lot of good analysis using an interesting and unique dataset. The $\mathrm{CO}$ and $\mathrm{CO} 2$ measurements look spot-on with the long-term ground monitoring network data, which is very encouraging since these airborne measurements can be tricky given the large background. With the suggested restructuring and a tighter focus on how the findings are more broadly applicable, the manuscript should be quite suitable to publish in ACP.

Interactive comment on Atmos. Chem. Phys. Discuss., https://doi.org/10.5194/acp-2019-70, 2019. 